\title{
Zusammenfassung und Forschungsfragen
}

Schulleitende und Lehrpersonen stehen vor der Herausforderung eines zunehmenden Gestaltwandels des Unterrichts: Ein ausschließlich eng geführter Klassenunterricht, in dem Lehrpersonen als reine Stoffvermittlerinnen und Stoffvermittler lehren, gilt kaum noch als probates didaktisches Lernsetting, um heterogenen Lerngruppen, digitalem Lernen oder der Leitidee der Kompetenzorientierung gerecht $\mathrm{zu}$ werden. Auch in einer sich wandelnden Schule bleibt jedoch das Ziel bestehen, kognitiv aktivierende, auf Verstehen ausgerichtete Lernprozesse anzuregen, in denen Schülerinnen und Schüler eigenständig und aktiv in einer Lerngemeinschaft wirken und sich als mitverantwortlich für das Erreichen der eigenen Lern- und Kompetenzziele erleben (Oelkers \& Reusser, 2008).

In zahlreichen Schulen u. a. in der Schweiz haben sich Schulleitende und Lehrpersonen deshalb dazu entschlossen, Jahrgangsklassen und/oder leistungsgetrennte Klassen zu durchmischen, Unterrichtsstunden in geführte und offene Phasen zu unterteilen und die Zeitstrukturen so anzupassen, dass geführte Unterrichtsphasen meist weniger lang und offenere Phasen länger als 45 Minuten andauern. Neben diesen veränderten organisationalen Schul- und Unterrichtsstrukturen wird auch die Aufgabenkultur weiterentwickelt und das Spektrum von Lehr- und Lernformen wie auch die Formen der Lernunterstützung werden erweitert (Reusser, 2019). Diese Entwicklungstätigkeiten zielen auf eine verstärkt personalisierte Gestaltung von Lehr- und Lernprozessen ab, in denen Lehrpersonen vor allem

- das Lernangebot an die personalen Voraussetzungen der Schülerinnen und Schüler anpassen,

- personale und soziale Kompetenzen aufbauen und Schülerinnen und Schüler ganzheitlich fördern,

- selbstgesteuertes Lernen auf eigenen Wegen ermöglichen, 
- kompetenzorientiertes Lernen zur persönlichen Sache machen und

- bildend und unterstützend wirken (siehe Abschnitt 3.2, u. a. Stebler et al., 2018).

Die damit einhergehenden Entwicklungstätigkeiten werden unter einer $u$ nterrichtszentrierten Schulentwicklung in Richtung personalisierten Lernens (uSpL) subsumiert. Das hierzu entwickelte uSpL-Modell (siehe Abschnitt 4.2) gründet auf der Zusammenführung von Theorien und Modellen der Schulentwicklung (u. a. Lozano et al., 2017; Maag Merki, 2017; Rolff, 2013) hinsichtlich der Entwicklung der Einzelschule als pädagogische Handlungseinheit (Fend, 1986) und der pädagogisch-psychologischen Lehr- und Lernforschung (u. a. Reusser, 2011). Weiterentwickelt wurde das uSpL-Modell während der Analyse der empirischen Daten der vorliegenden Forschungsarbeit. Aus diesem Grund ist das uSpL-Modell in erster Linie ein Analysemodell für Entwicklungsprozesse, kann allerdings darüber hinaus auch als heuristisches Modell zur Hypothesenbildung und zu deren empirischer Überprüfung eingesetzt werden.

Prozesse der Schul- und Unterrichtsentwicklung zielen auf verbesserte, multikriteriale Bildungswirkungen ab (fachliche und überfachliche Kompetenzen, motivationale Orientierungen etc.) (u. a. Bastian, 2007; Kiper, 2013; Klafki, 1985/2007; Maag Merki, 2016, 2018; Rolff, 2014). Allerdings wird in den meisten Publikationen zum Thema "Schulentwicklung“ das dahinterliegende Lernverständnis kaum definiert. Das uSpL-Modell hingegen setzt beim Lernverständnis an: Ausgehend von einem kognitiv- und soziokonstruktivistischen Lernverständnis (siehe Abschnitt 4.5.1.1) wird Unterricht gemäß dem Modell des didaktischen Dreiecks als (siehe Abschnitt 4.5.1.2) dreigliedrige Relation von Lehrperson, Lernenden (als Einzelpersonen oder als Gruppe) und Lerngegenstand beschrieben, woraus drei didaktischen Qualitätskulturen resultieren: die bildungsinhaltliche Ziel- und Stoffkultur (Was soll warum und wozu gelehrt werden?), die auf den Lehr- und Lernprozess bezogene Lehr- und Lernkultur (Wie und in welcher Prozessqualität soll gelernt und gelehrt werden?) und die kommunikativunterstützende Interaktions- und Unterstützungskultur (Womit und wodurch kann Lernen unterstützt, begleitet und ermutigt werden?). Kernzone didaktischen Handelns der Lehrperson sind entlang der drei Teilkulturen die inhalts-, prozess- und interaktionsbezogenen Handlungsaufgaben sowie die Steuerung der kognitiven, sozialen und motivational-emotionalen Dynamiken im Lehr- und Lerngeschehen in Abhängigkeit von den Lernenden und den Lerngegenständen (u. a. Reusser, 2009).

Entwicklungstätigkeiten für eine verstärkt personalisierte Gestaltung von Lehrund Lernprozessen zielen auf ebendieses didaktische Handeln ab. Jedoch stellt 
sich die Frage, welche Aspekte didaktischen Handelns am wirksamsten sind, um im Rahmen der Personalisierung verbesserte Bildungswirkungen zu erzielen (siehe Abschnitt 4.5.1.3). Eine mögliche Antwort auf diese Frage liefert die auf Aeblis (1983/2006) psychologischer Didaktik aufbauende Differenzierung zwischen Oberflächen- und Tiefenstruktur des Unterrichts (Reusser, 1999). Während Erstere die Handlungsstrukturebene u. a. mit dem variablen Spiel aus Methoden, Sozial- und Inszenierungsformen umfasst, bezieht sich die Tiefenstruktur auf psychologische Prozesse und Merkmale des Lehrens und Lernens, die dem Unterricht als psychologisch-didaktische Qualitätsmerkmale zugrunde liegen (Reusser \& Pauli, 2010b), und somit auf die Frage, inwiefern es die Lehrperson gelingt, Schülerinnen und Schüler zu verstehensbezogenen Lernprozessen anzuregen (Aebli, 1983). Dem uSpL-Modell liegt diesbezüglich die Annahme zugrunde, dass Entwicklungsprozesse die Bildungswirkungen dann positiv beeinflussen, wenn die Gestaltung von Lernumgebungen in ihrer Umsetzungsqualität die Tiefenstrukturebene des Lernens erreicht, das heißt nachhaltiges und verstehensbezogenes Lernen auszulösen vermag.

Neben einem kognitiv- und soziokonstruktivistischen Lernverständnis geht das uSpL-Modell von einer akteurs- und handlungstheoretischen Perspektive aus: Einbezogen werden die Mitwirkenden und deren Tätigkeitsfelder (Entwicklungsdimensionen), wobei die Mitwirkenden in zwei Gruppen unterteilt werden, nämlich zum einen in die innerschulischen Akteursgruppen (siehe Abschnitt 4.7) - Schulleitende, Lehrpersonen und Schülerinnen und Schüler -, die direkt den Entwicklungsprozess steuern und Entwicklungsimpulse rekontextualisieren (Fend, 2008b), und zum anderen in außerschulische Akteursgruppen (siehe Abschnitt 4.8), das heißt Schulbehörden, Beratende, Eltern und Mitglieder in schulischen Netzwerken.

Für die in der vorliegenden Arbeit im Mittelpunkt stehende Entwicklungsdimension der Unterrichtsentwicklung in Richtung einer personalisierten Gestaltung von Lehr- und Lernprozessen (siehe Abschnitt 4.5.1) sind das bereits erwähnte didaktische Dreieck sowie die Unterscheidung zwischen Merkmalen der Oberflächen- und der Tiefenstruktur des Unterrichts zentral. Weitere berücksichtigte Entwicklungsdimensionen sind die Folgenden:

- Entwicklung organisationaler Strukturen: Zentrale Elemente sind die räumliche und die zeitliche Strukturierung, die Zusammensetzung der Lerngruppen sowie Team- und Kooperationsstrukturen (siehe Abschnitt 4.5.2).

- Entwicklung der Kooperation zwischen Lehrpersonen: Diesbezüglich zeigen Forschungsergebnisse auf, dass unterrichtsbezogene und ko-konstruktive Kooperation eine im Hinblick auf den Erfolg des Entwicklungsprozesses und 
auch bezüglich eigener Professionalisierungsprozesse eine wirkungsvolle Form der Zusammenarbeit darstellen kann. Potenziale dieser Kooperation ergeben sich nicht nur in den gemeinsamen Entwicklungstätigkeiten, sondern auch in der alltäglichen Unterrichtsarbeit mit Konzepten personalisierten Lernens (siehe Abschnitt 4.5.3).

- Professionalisierung des Schulpersonals und Personalrekrutierung: Das Schulpersonal kann durch schulinterne und schulexterne Fort- und Weiterbildung und ebenso durch eine ko-konstruktive und unterrichtsbezogene Kooperation professionalisiert werden. Des Weiteren lassen sich geeignete Lehrpersonen durch gezielte Rekrutierung gewinnen (siehe Abschnitt 4.5.4).

- Entwicklung eines gemeinschaftlichen Schullebens: Diese Dimension bezieht sich auf das gemeinsame „Unterwegssein“ über den Unterricht hinaus und das Zusammengehörigkeitsgefühl unter den Schülerinnen und Schülern und Lehrpersonen (siehe Abschnitt 4.5.5).

Im uSpL-Modell wird neben den Tätigkeitsfeldern und den bedeutsamen Akteursgruppen auch die Prozessebene dargestellt (siehe Abschnitt 4.6): Ein Entwicklungsprozess wird einerseits durch die drei Phasen der Initiation, der Implementation und der Institutionalisierung (Giaquinta, 1973) und andererseits durch eine Wechselwirkung von Strukturmomenten und konkreten Handlungen (Giddens, 1988) bestimmt. Einen weiteren Bereich im Entwicklungsprozess bilden die Rahmenbedingungen (siehe Abschnitt 4.9). Dazu gehören die Zusammensetzung der Schülerinnen und Schüler (Einzugsgebiet der Schule), finanzielle Ressourcen und die für die Entwicklungstätigkeiten verfügbare Zeit sowie Schulform und Trägerschaft. Im letzten Bereich, der sich auf die Metaziele bezieht, zielt die personalisierte Gestaltung von Lehr- und Lernprozessen auf verbesserte Lernfortschritte der Schülerinnen und Schüler bei fachlichen und überfachlichen Kompetenzen, motivationalen Orientierungen, Interessenbildung, Einstellungen, erzieherischen und sozialisatorischen Aspekten etc. ab (siehe Abschnitt 4.10).

Zusammenfassend dient das uSpL-Modell dazu, die folgenden Fragen in Bezug auf einen Entwicklungsprozess in Richtung personalisierten Lernens zu klären:

- WARUM? = Auslöser;

- WER? = Mitwirkende/Akteursgruppen;

- WAS? = Entwicklungsdimensionen;

- $\mathrm{WIE}$ ? = Prozessverlauf;

- WOMIT? = Rahmenbedingungen und Prozesssteuerung;

- WOHIN? = Metaziele 
Das uSpL-Modell stellt somit einen Versuch dar, die Komplexität eines Entwicklungsprozesses zu modellieren. Die Komplexität der Modellierung erwächst u. a. aus den verschiedenen, miteinander interagierenden Akteurinnen und Akteuren, die mit unterschiedlichen Zielen und Intentionen den Entwicklungsprozess hinsichtlich verschiedener Entwicklungsdimensionen gemeinsam gestalten. Der rezipierte Forschungsstand zu personalisiertem Lernen zeigt, dass zu den einzelnen Themenbereichen teilweise viele empirische Befunde vorliegen. Allerdings sind in einigen Themenbereichen, zum Beispiel zum Mitwirken außerschulischer Akteursgruppen, zugleich umfangreiche Forschungsdesiderate festzustellen. Darüber hinaus lässt sich in der einschlägigen Literatur keine Studie finden, in welcher der Entwicklungsprozess in seiner Gesamtheit systematisch und längsschnittlich untersucht wurde, um die Fragen zu beantworten, WARUM sich die Schulen in Richtung personalisierten Lernens weiterentwickeln, WOHIN sie sich weiterentwickeln und WER im Entwicklungsprozess mit wem, WIE und WOMIT im Hinblick auf WAS kooperiert.

Dieses Forschungsdesiderat wird mit der ersten Forschungsfrage bearbeitet:

1. Forschungsfrage: Wie, aus welchem Anlass, mit welchen Mitteln, mit welchem Ziel und unter Bearbeitung welcher Themen und Gegenstände gestalten Schulleitende und Lehrpersonen ihre unterrichtszentrierte Schulentwicklung in Richtung personalisierten Lernens?

Zur Klärung dieser Frage wird im ersten Teil von Studie 1 der Entwicklungsprozess von elf Schulen des perLen-Projekts im dreijährigen Erhebungszeitraum (20122015) anhand einer Analyse von teilstrukturierten Leitfadeninterviews beschrieben. Dabei wird den folgenden Teilfragen nachgegangen:

- Teilfrage 1.1: Welche Rahmenbedingungen weisen die elf Schulen auf?

- Teilfrage 1.2: Wann wurde in den Schulen damit begonnen, das jeweilige Konzept personalisierten Lernens zu entwickeln und einzuführen?

- Teilfrage 1.3: Wie sind Lernräume, Lerngruppen, Lernzeiten und weitere organisatorische Merkmale der Konzepte personalisierten Lernens strukturiert?

- Teilfrage 1.4: Wie entwickeln Schulleitende und Lehrpersonen innerhalb des dreijährigen Erhebungszeitraums ihr jeweiliges personalisiertes Unterrichtskonzept weiter?

- Teilfrage 1.5: Welche langfristigen Ziele (Metaziele) der unterrichtszentrierten Schulentwicklung in Richtung personalisierten Lernens werden genannt?

Im zweiten Teil von Studie 1 stehen die Gemeinsamkeiten in der Prozessgestaltung aller elf Schulen im Zentrum, das heißt die Frage, an welchen Gegenständen 
Schulleitende und Lehrpersonen arbeiten, um Konzepte personalisierten Lernens weiterzuentwickeln:

- Teilfrage 1.6: Welche Entwicklungsgegenstände lassen sich aufbauend auf dem Kategoriensystem der inhaltsanalytischen Datenanalyse (Studie 1/Teil 1) extrahieren?

- Teilfrage 1.7: Welche Entwicklungsgegenstände werden von Lehrpersonen und Schulleitenden aller Schulen für die Zeit vor oder/und für die Zeit während des Erhebungszeitraums beschrieben?

Wie bereits ausgeführt, wird im uSpL-Modell angenommen, dass Entwicklungstätigkeiten in erster Linie auf verbesserte Lernerfolge der Schülerinnen und Schüler abzielen (u. a. Bastian, 2007; Kiper, 2013; Klafki, 1985/2007; Maag Merki, 2016, 2018; Rolff, 2014). Diesbezüglich versprechen besonders diejenigen Entwicklungstätigkeiten eine hohe Wirksamkeit, die auf die Tiefenmerkmale des Unterrichts ausgerichtet sind, weil damit die psychologischen Tiefenschichten des Lernens gezielt beeinflusst werden können. Gleichwohl liegen derzeit, mit Ausnahme der Teilstudie von Meissner et al. (2016) aus dem WissGem-Projekt, keine Studien im Kontext personalisierten Lernens vor, die Entwicklungstätigkeiten hinsichtlich der Tiefenmerkmale von Unterricht untersuchen. Dieses Desiderat wird in Studie 2 mit den folgenden drei Forschungsfragen bearbeitet:

2. Forschungsfrage (Studie 2/Teil 1): Welchen Dimensionen und Merkmalen auf zwei Qualitätsebenen des Unterrichts (Oberflächen- und Tiefenstrukturebene) personalisierten Lernens lassen sich die identifizierten Entwicklungstätigkeiten zuordnen?

3. Forschungsfrage (Studie 2/Teil 2): Gibt es eine Beziehung zwischen der Selbsteinschätzung des Entwicklungsstandes der Schulen und dem von ihnen verfolgten Verständnis von Lehr- und Lernqualität?

\section{Forschungsfrage (Studie 2/Teil 3): Zeigen sich dabei Muster, nach denen sich die Schulen gruppieren lassen?}

Zuerst werden die Dimensionen personalisierten Lernens als empirisches Analysewerkzeug eingesetzt, um deren Vorkommen in den berichteten Entwicklungstätigkeiten zur Unterrichtsentwicklung zu analysieren (Studie 2/Teil 1):

- Teilfrage 2.1: Welche Dimensionen personalisierten Lernens (siehe Abschnitt 3.2) lassen sich in den Interviewaussagen zur Unterrichtsentwicklung wiederfinden? 
- Teilfrage 2.2: Wie lassen sich die Dimensionen personalisierten Lernens anhand der Interviewaussagen beschreiben und konkretisieren?

Anschließend wird im 2. Teil von Studie 2, wie auch in der Studie von Meissner et al. (2016), untersucht, welche Interviewaussagen aus den Codierungen von Studie 2/Teil $1 \mathrm{zu}$ Merkmalen der Oberflächenstruktur und welche Aussagen zu Merkmalen der Tiefenstruktur zugeordnet werden können und in welchem Anteilsverhältnis diese stehen. Im Unterschied zur Studie von Meissner et al. (2016) werden in der vorliegenden Analyse jedoch nicht die einzelnen Interviewaussagen ausgezählt, sondern es erfolgt eine Zusammenfassung von inhaltlich ähnlichen Aussagen (Summary). Dies hat methodische Gründe einer reliableren Quantifizierung von Interviewaussagen (siehe Abschnitt 6.4.2).

- Teilfrage 2.3: Welche Aspekte der Oberflächen- und der Tiefenstruktur von Unterricht werden in den Interviewaussagen zur Unterrichtsentwicklung thematisiert?

- Teilfrage 2.4: In welchem prozentualen Verhältnis stehen die Anteile der Interviewaussagen, die der Qualitätsebene der Oberflächenstruktur des Unterrichts zugeordnet werden können, zu den Anteilen der Interviewaussagen, die der Qualitätsebene der Tiefenstruktur von Unterricht zugeordnet werden können in jeder der elf Schulen?

Im Kontext eines Verständnisses von Einzelschulen als pädagogische Handlungseinheiten (Fend, 1986) wird erwartet, dass sich die Schulen in den prozentualen Anteilsverhältnissen voneinander unterscheiden, da Schulleitende und Lehrpersonen Entwicklungsimpulse und -vorhaben in Abhängigkeit von verfügbaren beruflichen Kompetenzen, finanziellen Ressourcen, der Heterogenität der Schülerinnen und Schüler, der Kooperationsqualität im Team und weiteren Handlungsbedingungen unterschiedlich rekontextualisieren,.

Ein Faktor, welcher den Erfolg der Adaption von Entwicklungsimpulsen an die eigenen Handlungsroutinen $\mathrm{zu}$ einem gewissen Grad misst, ist der selbst eingeschätzte Stand der Schulentwicklung. Im dritten Erhebungsjahr wurden Schulleitende und Lehrpersonen deshalb gefragt, wie sie den Entwicklungsstand ihrer Schule auf einer Skala zwischen ,gar nicht gut unterwegs" und ,hervorragend unterwegs“" einschätzen. Diesbezüglich wird angenommen, dass der Entwicklungsstand mit dem prozentualen Anteil der Tiefenstrukturmerkmale von Unterricht positiv zusammenhängt. Diese Annahme gründet auf der Überlegung, dass Lehrpersonen mit ihren Entwicklungstätigkeiten zufrieden sein können, wenn sie es schaffen, mit ihren Konzepten personalisierten Lernens kognitive, motivational-emotionale 
und soziale Dynamiken des Lernens positiv zu beeinflussen, respektive wenn sie sich dieser Tiefenstrukturebene des Lehrens und Lernens bewusst sind, sie erfassen und kompetent gestalten können, um an die psychologischen Tiefenschichten des Lernens der Schülerinnen und Schüler heranzukommen und verstehensbezogenes Lernen auszulösen. Auf der Grundlage dieser Überlegung wurde die folgende Frage formuliert (Studie 2/Teil 3):

- Teilfrage 2.5: Zeigt sich ein Zusammenhang zwischen den Anteilen zusammengefasster Interviewaussagen (Summaries), die der Qualitätsebene der Tiefenstruktur des Unterrichts zugeordnet werden können, und der Einschätzung des Entwicklungsstands einer jeden Schule?

Des Weiteren ermöglicht es die erwartete Varianz im prozentualen Verhältnis, die Schulen in Clustern zu gruppieren. Theoretisch lassen sich ausgehend von den zwei Merkmalen vier Cluster ableiten: Cluster 1, bei dem die Werte in beiden Bereichen hoch sind, Cluster 2 mit niedrigeren Werten in der Tiefenstruktur und höheren Werten in der Einschätzung des Entwicklungsstands, Cluster 3 mit umgekehrten Verteilungen, das heißt hohe Werte bezüglich der Tiefenstruktur und niedrige Werte beim Entwicklungsstand, und Cluster 4 mit niedrigeren Werten bei beiden Merkmalen. In Anbetracht eines möglichen positiven Zusammenhangs würden Cluster 1 und Cluster 4 zu erwarten sein. Inwiefern sich diese Gruppierung empirisch belegen lässt und wie sich diese charakterisiert, wird mit den letzten zwei Teilfragen bearbeitet:

- Teilfrage 2.6: Lassen sich die Schulen anhand ihrer jeweiligen Anteile an Summaries, die der Qualitätsebene der Tiefenstruktur des Unterrichts zugeordnet werden können, und ihrer Einschätzung des Entwicklungsstands gruppieren?

- Teilfrage 2.7: Wie lassen sich mögliche Entwicklungsgruppen (Cluster) charakterisieren? 
Open Access Dieses Kapitel wird unter der Creative Commons Namensnennung 4.0 International Lizenz (http://creativecommons.org/licenses/by/4.0/deed.de) veröffentlicht, welche die Nutzung, Vervielfältigung, Bearbeitung, Verbreitung und Wiedergabe in jeglichem Medium und Format erlaubt, sofern Sie den/die ursprünglichen Autor(en) und die Quelle ordnungsgemäß nennen, einen Link zur Creative Commons Lizenz beifügen und angeben, ob Änderungen vorgenommen wurden.

Die in diesem Kapitel enthaltenen Bilder und sonstiges Drittmaterial unterliegen ebenfalls der genannten Creative Commons Lizenz, sofern sich aus der Abbildungslegende nichts anderes ergibt. Sofern das betreffende Material nicht unter der genannten Creative Commons Lizenz steht und die betreffende Handlung nicht nach gesetzlichen Vorschriften erlaubt ist, ist für die oben aufgeführten Weiterverwendungen des Materials die Einwilligung des jeweiligen Rechteinhabers einzuholen. 